\title{
Preliminary study on fault detection using artificial neural network for water-cooled reactors
}

\begin{abstract}
In the PUSPATI TRIGA reactor (RTP), many variables and instruments need to be monitored to make sure it is functioning and running accordingly. The late detection of faults may result in accidents and affect workers' safety and health. Therefore, an intelligent fault detection system is needed to detect faults in the process plant and alert for any safe point breach. This work was carried out to discover the use of an artificial neural network (ANN) to model and develop a fault detection programme in the RTP cooling system. Using actual data from the reactor to train the multilayer network model with backpropagation algorithm. Referring to the real data from the reactor, the simulation results demonstrate a good correlation between the proposed model using ANN and the real plants with a residual mean of below $1 \%$. The preliminary results for fault detection show that ANN was able to predict the value of failure in residual factor by comparing the normal state and fault state of the plant. The proposed model using ANN method proofed that it could quickly diagnose the single fault and perform for any given failure. The research outcome could contribute to the improvement in frontier technologies and advanced manufacturing in Malaysia.
\end{abstract}

Keyword: Artificial neural network; Cooling system; Structures; Systems and components; Fault detection; Residual 